\title{
CORRESPONDENCE
}

Vaccine against swine influenza

F E André, MrCPATH, and others. . . . . 1527

Multiple sclerosis among immigrants

J F Kurtzke, FACP . . . . . . . . . . . . . 1527

Folate-responsive neuropathy

M C Bateson, MRCP; J Runcie, FRCPGLAS,

and $M$ Manzoor, MB............ 1528

Vaginal candidosis

G R Daniel, MB; R A Sparks, MRCoG; C S

Good, FRCS; R Pumpianski, $M D$, and

$S$ Ganor, MD................ 1528

Fetal activity and fetal wellbeing

P R B Pedlow, FRCog; D D Mathews,

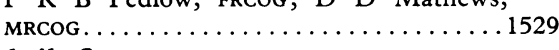

Febrile fits

S Livingston, MD, and Lydia L Pauli, MD . . 1530

Perhexiline maleate in angina pectoris

J D F Lockhart, MB, and H C Masheter, MB 1530

Intermittent claudication

M Thomas, FRCS, and C R G Quick, FRCS. . 1531
Laparoscopy explosion hazards with nitrous oxide

G B Drummond, FFARCs, and D B Scott, FFARCS. . . . . . . . . . . . . . . . 1531

Prevention of coronary heart disease

M A Crawford, PHD; José A Day, MB . . . 1532

Fluid therapy in malaria

V Sitprija, FACP................ 1532

Unexplained hepatitis following halothane

W K Slack, DA................... 1532

Five separate malignancies in one patient

P A Jones, frCs . . . . . . . . . . . . 1533

Hazards of monocomponent insulins

C $M$ Kesson, MrCP.................. 1533

Computer-held medical records

C J Bulpitt, MRCP, and others. . . . . . . 1533

Incidence of dermographism

R F Q Johnson, PHD ........... 1533

Cigarette consumption

M Daube.................... 1534
Occult abdominal injury

B J Pardy, FRCS........

P R V Tomson, FRCGP; April G L Kay,

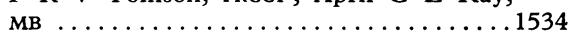

Status of medical laboratory scientists

A D Farr, fimls................. 1534

Doctors and administrators

R J Luck, FRCs, and others........... 1534

Ophthalmic medical practitioners

W J C C Rich, FRCS. . . . . . . . . . . . . 1535

Campaign for Independence in Medicine

J R Saunders, FRCOG . . . . . . . . . . 1535

Points from letters Inlet or outlet? (D I Grieve); Intermittent claudication (T Chakera); Visual fields in coma? (D W W Bullimore); Nostalgia: a vanished disease (J H S Pettit); Impact therapy for chronic bronchitis (J B Tracey); Flupenthixol (N P Mann); Ocular complications of ethambutol (R S Bartholomew); Profession and Government (P R J Vickers) 1535
Correspondents are urged to write briefly so that readers may be offered as wide a selection of letters as possible. So many are being received that the omission of some is inevitable. Letters should be signed personally by all their authors.

\section{Vaccine against swine influenza}

SIR,-WHO influenza experts have recently recommended the inclusion of the $\mathrm{A} / \mathrm{New}$ Jersey/8/76 $\left(\mathrm{Hsw}_{1} \mathrm{~N}_{1}\right)$ virus in inactivated vaccines for $1976-7 .{ }^{1}$ That decision was prompted by the small outbreak caused by this strain in a military training camp in the USA which led President Ford to advocate a Government-sponsored mass immunisation campaign. This unprecedented measure was dictated by the current low level of specific immunity of most people younger than 50 against this virus, which is antigenically related to the swine influenza virus thought to have been responsible for the catastrophic 1918 pandemic (Spanish "flu").

We produced in our laboratories a small lot of $\beta$-propiolactone-inactivated wholevirion vaccine containing $400 \mathrm{IU}$ of $\mathrm{A} / \mathrm{New}$ Jersey/8/76 (X-53 $\left(\mathrm{Hsw}_{1} \mathrm{~N}_{1}\right), 400 \mathrm{IU}$ of A/Victoria/3/75 (X-47 $\left(\mathrm{H}_{3} \mathrm{~N}_{2}\right)$, and $360 \mathrm{IU}$ of $\mathrm{B} /$ Hong Kong $/ 8 / 73$ per dose in order to vaccinate company employees who are, or could become, engaged in influenza vaccine research, production, and control. The opportunity was taken to investigate the serological response to one dose of this new vaccine.

Blood samples were obtained about one month before and 19 days after vaccination from a total of 40 vaccinees (14 women (19-57 years) and 26 men (22-57 years)). Only four had not previously received influenza vaccine. Antibody titrations are still in progress but we should like to report our findings on the serum haemagglutination inhibition (HI) antibody response to the $\mathrm{A} / \mathrm{New}$ Jersey/8/76 component in the vaccine. To our knowledge these are the first to become available. Each HI titre is the geometric mean of two independent titrations by a standard technique using cholera filtrate-treated serum and 3 haemagglutinating units of $\mathrm{A} / \mathrm{New}$ Jersey antigen. The results are summarised in the table.

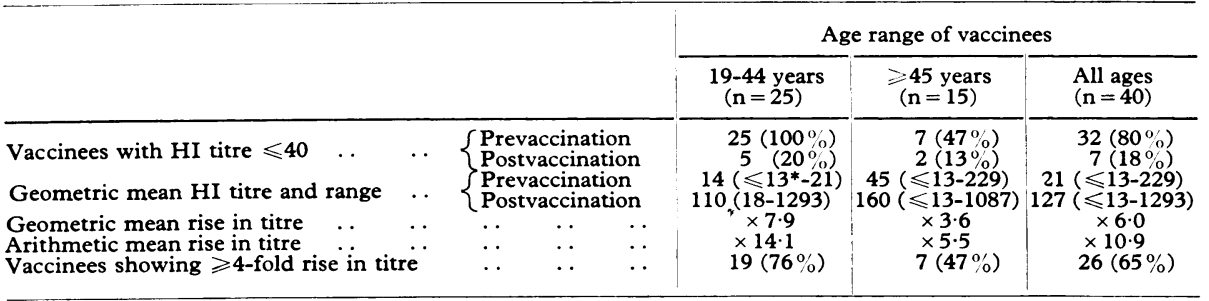

*An undetectable antibody HI titre $(\leqslant 13)$ was assigned a value of 13 for calculations.

It is clear that $400 \mathrm{IU}$ of $\mathrm{A} / \mathrm{New}$ Jersey/8/76 vaccine gave a good homologous serum HI antibody response after a single dose in vaccinees above 45 years, 11 of whom had pre-existing antibodies to the strain owing to the fact that swine-like viruses circulated among humans up to about the early 1930s. The response was equally good, if not better, in younger vaccinees who are unlikely to have been exposed to swine-influenza viruses. Three of these had low prevaccination titres and we suspect that these reflect cross-reacting antibodies. Of 26 vaccinees with undetectable HI titre $(\leqslant 13)$ before vaccination, 20 reached a titre of $\geqslant 40$ after vaccination. Only one person showed no rise in serum HI titre against $\mathrm{A} / \mathrm{New}$ Jersey after vaccination. Thus we conclude that this dose of vaccine will probably confer adequate immunity against the $\mathrm{A} / \mathrm{New}$ Jersey virus, at least to all adult age groups.

F ANDRÉ

$\mathrm{J}$ W LEYENHORST J C SEKET

D ZellenRATH

Philips-Duphar BV,

Weesp, Holland

${ }^{1}$ World Health Organisation, Weekly Epidemiological Record, 1976, 51, No 6.

\section{Multiple sclerosis among immigrants}

SIR,-Once again I must disagree with my good friend, Dr Geoffrey Dean. In their admirable study on multiple sclerosis (MS) among immigrants in greater London (10 April, p 861) Dr Dean and his colleagues conclude that "those who migrate from lowrisk countries to London, a high-risk area, keep their low risk of developing the disease; 
immigrating to England does not seem to increase their risk of MS." I submit that the data presented do not speak to the question of the same versus a higher risk among such immigrants. At face value they would indicate only that there are notably fewer such patients admitted to hospital than expected for native Britons. Whether this reflects more than or the same number affected as if they had stayed at home is not definable.

But even the question as to the numbers observed in relation to those expected is not well satisfied here, in my opinion. I really doubt if crossing the Irish Sea alters appreciably one's chances of having MS, and yet only 168 such patients were ascertained while 225 were expected. Whether this represents input bias or an inappropriate expected number (or both) is undefined. If this be so for the Irish, how much more might this be true of the "new Commonwealth" immigrants? (As an aside, for the colonies at least, definition of specific countries incorporated into the New Britannia would have been helpful.)

The use of a 1971 age distribution for 1966 immigrants may well be questioned because of the drastic increase in numbers, there being over twice as many immigrants in 1971 as in 1961. There is a more important aspect to this increase, however. New Commonwealth immigrants to England from Asia numbered 270000 in 1961 and 618000 in 1971; from Africa 43000 and 158000 respectively. Thus, one would expect that the majority of such immigrants had been in England less than five years. This raises the practical problem of expectations (by age and sex) from 13 years of hospital admissions versus those from five years or so. This is not completely refuted by limitation to "first admissions of newly diagnosed cases." Some correction perhaps should have been employed for person-years of exposure to the chance of admission.

In terms of the disease itself the rapid increase in the population at risk poses yet other and more important problems. Is five years or so a sufficient period to express an increased risk of MS, as must have been the case for more than half the series? Further, is "exposure" from age 50 to 54 or from age 5 to 9 meaningful in terms of MS ? Also, can lifetime "exposure" among the British be equated with shorter "exposure" among immigrants? The question of risk of MS in migrant populations gets very involved, being dependent not only on a sufficiency of people who change their residence from one risk area to another but also on their ages at immigration, their length of stay in the new land, and their ages at prevalence day. It is only to this last point that the expectations of Dr Dean and his colleagues are really addressed. Another problem further to confound the issue is the apparent racial predilection for MS, regardless of geography: MS seems the white man's burden. ${ }^{1}$

There is evidence from mortality data that moving from low-risk to high-risk MS regions does increase the risk of dying from MS. ${ }^{2}$ Unpublished data from a large series of US veterans with $M S$ versus military controls also support the concept of an increased risk of MS among southern-born who moved north before entry into service. We have also some information to the same effect from an unfortunately small cohort of Vietnamese who moved to France. However, these works too cannot be taken as definitive, and the question of the fate of migrants from low- to high-risk areas in terms of MS, I believe, perhaps still warrants the Scottish verdict of "not proven."
The study of Dr Dean and his colleagues is indeed a formidable one. Certainly the massive deficit of cases found does not seem readily explicable merely by input bias. There may be further gold to be mined therein. One simple approach would be to look only at the numerator data and to compare immigrants from the varying risk area, but according to age at immigration versus age of onset and age and calendar year of London diagnosis plus race. Distributions of the denominator by age and calendar year of immigration, by country, would also be informative.

Department of Neurology and

Community Medicine

Georgetown University School of Medicine, and

Neurology Service,

Veterans Adminis ' Kurtzke, J F, Acta Neurologica Scandinavica, 1975,

' Kurtzke, J F, et al, Neurology, 1971, 21, 1186

***As we understand it, the "new Commonwealth" consists of those countries of the old British Empire, too numerous to detail, that have achieved independence since 1945 and have remained within the Commonwealth. -ED, $B M \mathcal{F}$

\section{Folate-responsive neuropathy}

SIR,-Two challenging propositions are put forth in the article by Drs M Manzoor and J Runcie (15 May, p 1176). They contend that folate deficiency causes a specific nf urological disorder "clinically indistinguishable from subacute combined degeneration of the spinal cord" and that folic acid therapy reverses the neuropathy. If proved, then these observations would offer an important opportunity in the management of otherwise intractably disabled patients. Unfortunately, the hypotheses have not been rigorously tested and require more evidence before acceptance.

The 10 patients recorded were considered to be folate deficient on the results of the activity of Lactobacillus casei stimulated by their serum. In none was bone marrow aspiration recorded and only five had a macrocytic anaemia. No comment was made about the possible effect of antibacterial therapy on the microbiological assay system. No results are presented for red cell folate assay, and this more reliable test is surely essential if serious disease is to be attributed to chronic folate deficiency.

It could easily be that inadequate nutrition was secondary to the immobility described. If conclusions are to be drawn about the causative role of folate deficiency in severe neuropathy, then a minimum requirement should be estimation of serum and red cell folate activity in a matched group with comparably severe disability of known aetiology, such as Parkinson's disease.

The conclusion that therapy with folic acid lessened the observed neuropathy could be sustained only by a double-blind controlled trial against placebo. It is exceptionally difficult to single out one facet of medical care as being of overriding importance. It would be regrettable if geriatricians influenced by this article relaxed the effort to remobilise patients because of undue reliance on vitamin therapy of unproved benefit.

It hardly needs restating that folic acid therapy in undiagnosed vitamin $B_{12}$ deficiency is potentially disastrous.

MaLColm C Bateson

Department of Medicine,

Ninewells Hospital,

Dundee

$*_{*}^{*}$ We sent a copy of this letter to Drs Manzoor and Runcie, whose reply is printed below.

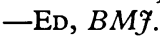

SIR,-We are grateful to Dr Bateson for his letter. The views contained therein encapsulate those clinical attitudes which we are anxious to challenge. The definition of folate deficiency states is difficult and at present imprecise. The validity of a low serum folate level is not in question, but rather the interpretation of its clinical significance. In a confused patient with red cell macrocytosis and a florid neuropathy, in our view, little clinical acumen is required to decide this. An important rider in patients with very low serum folate values is the effect of recent antibiotic therapy on this. None of our patients were so treated for at least 10 days prior to folate measurement.

The reference to immobility we find puzzling. The neuropathy we described is not a feature of severe, completely immobilising stroke, for example. Furthermore, intensive physiotherapy in our patients in the absence of concurrent folate administration is without value.

There is no place for the superficially attractive double-blind trial of tolate against placebo in a neurological disorder which takes many months and possibly some years to develop and during which increasing and perhaps irreparable damage to the nervous system occurs.

We would agree wholeheartedly that the indiscriminate use of folate in obscure neurological disease is indefensible. This danger is completely avoided by measuring the serum $B_{12}$ and folate levels together and never singly. The use of folate in such circumstances when the serum $B_{12}$ is known to be normal is quite different and a clinical trial of such is eminently justifiable.

JOHN RUNCIE

MOHINDRA MANZOOR

University Department of

Geriatric Medicine,

Stobhill General Hospital,

Glasgow

\section{Vaginal candidosis}

SIR,-In your leading article on this subject (14 February, p 357) it is stated that "there is no evidence that the fungus [Candida albicans] develops any resistance to antifungal agents (with the one exception of amphotericin)." As manufacturers of amphotericin we are interested in this last comment since, according to our records and published evidence, we know of no substantiated clinical reports of resistance to the drug.

The early in-vitro studies of Littman $e t$ al ${ }^{1}$ failed to demonstrate resistance to amphotericin in the strains of $C$ albicans tested; and Athar and Winner" tested some 2015 clinical isolates of candida and found none naturally resistant. It was possible to produce in-vitro resistance by numercus passages, but in such cases the degree of pathogenicity was greatly reduced. ${ }^{2-5}$ As Hamilton-Miller ${ }^{3}$ stated as recently as 1974, "Allowing for the well-known 